INPLASY

PROTOCOL

To cite: Nobre et al. Uses and effects of self-assessment procedures in the analysis of student's performance in

Physical Education classes: A Systematic Review. Inplasy protocol 202150015. doi: 10.37766/inplasy2021.5.0015

Received: 03 May 2021

Published: 04 May 2021

Corresponding author:

Paulo Renato Nobre

prnobre@fcdef.uc.pt

Author Affiliation:

Research Unit for Sport and Physical Activity (CIDAF, UID/ PTD/04213/2019), University of Coimbra, 3040-248 Coimbra, Portugal.

Support: None.

Review Stage at time of this submission: Data analysis.

Conflicts of interest:

None declared.

\section{Uses and effects of self-assessment procedures in the analysis of student's performance in Physical Education classes: A Systematic Review}

Nobre, PR1; Fachada, MÂ2; Abreu, $\mathrm{A}^{3}$; Sarmento, H4; Silva, FM5; Soares, CM6; Cristovão, AL7; Teixeira, $A^{8}$; Ferreira, JP9 .

Review question / Objective: The aim of this systematic review is to summarize the published evidence on the use of selfassessment procedures in Physical Education and to identify the magnitude of the effects produced by these assessment practices in Physical Education (PE) classes, over the last ten years.

Condition being studied: Self-assessment uses and observed effects in Physical Education school settings.

Information sources: This systematic review comprises an extensive search of eight main databases: EBSCO, ProQuest, PubMed, Scopus, Taylor and Francis and Web of Science. Articles in Portuguese language were searched in Capes and Scielo databases. Searching process for relevant articles is limited to past ten years, from 2010 to 2020, employing the search string "physical education" AND "self-assessment" within the fields "article," "keywords," and "abstract". Supplementary analysis of the reference lists of the extracted articles was carried out manually to find potential articles, not electronically identified.

INPLASY registration number: This protocol was registered with the International Platform of Registered Systematic Review and Meta-Analysis Protocols (INPLASY) on 04 May 2021 and was last updated on 04 May 2021 (registration number INPLASY202150015).

\section{INTRODUCTION}

Review question / Objective: The aim of this systematic review is to summarize the published evidence on the use of selfassessment procedures in Physical Education and to identify the magnitude of the effects produced by these assessment practices in Physical Education (PE) classes, over the last ten years.

Rationale: Recent systematic reviews published about the use of peer and selfassessment procedures during evaluation 
in Physical Education revealed a shortage of research information about the peer and self-assessment process use in general, but specifically in school environment (Bores-García et al, 2020; Otero-Saborido et al, 2021). The need to broaden knowledge about the effectiveness for the use of different self-assessment tools and procedures to evaluate student's individual performance in PE classes is, therefore, justified and needed. Thus, it's important to understand whether this shortage of information, based in a very limited number of studies, means that these practices are not used by the PE teachers or if they exist but have not been explored as a major topic of research. In this systematic review we intend to summarize and report evidence of the use of different selfassessment tools and procedures with regular $P E$ students and $P E$ teacher training classes and to analyze the observed effects produced by this type of assessment practices in Physical Education (PE).

Condition being studied: Self-assessment uses and observed effects in Physical Education school settings.

\section{METHODS}

Search strategy: A systematic review of research published about the use of selfassessment procedures in Physical Education over the last ten years is performed in accordance with Preferred Reported Items for Systematic Reviews and Meta-analysis (PRISMA) guidelines. The search was conducted in the following eight electronic databases: CAPES, EBSCO, ProQuest, PubMed, Scielo, Scopus, Taylor and Francis and Web of Science, with access made between 15th december 2020 and 19th february 2021, using the advanced meta-search option in which original articles of observational studies, cross-sectional studies and qualitative studies published in Portuguese and English language between January 2010 and December 2020. The search strategy combined the following key terms and search indexed descriptors to refine the data: "physical education" and "self- assessment", using the AND command within the fields "article," "keywords," and "abstract". Portuguese correspondent keywords, "educação física" and "autoavaliação" were used for the search in Portuguese language (Brazil and Portugal). Papers that met the inclusion criteria were considered to be applicable to the review topic and retrieved for further assessment of methodological quality.

Participant or population: PE teachers and PE students; pre-service PE teachers.

Intervention: Self-assessment of students.

Comparator: Self-assessment procedures in PE regular school classes and PE teacher training classes. Self-assessment observed effects in PE regular school classes and PE teacher training classes.

Study designs to be included: Studies that meet publication criteria, qualitative or quantitative.

Eligibility criteria: The inclusion criteria used to determine which articles would be selected for the present study were: (a) empirical, observational, cross-sectional or qualitative studies published in a peerreviewed indexed journal; (b) studies reporting Physical Education selfassessment procedures in school settings; (c) availability of a full-text version of the article; (d) studies published in English or Portuguese language between January 2010 and December 2020. The exclusion criteria for articles were: (a) duplicated articles; (b) articles not published in indexed journals; (c) articles in languages other than English or Portuguese, and (d) Articles using self- assessment in contexts other than PE.

Information sources: This systematic review comprises an extensive search of eight main databases: EBSCO, ProQuest, PubMed, Scopus, Taylor and Francis and Web of Science. Articles in Portuguese language were searched in Capes and Scielo databases. Searching process for relevant articles is limited to past ten years, from 2010 to 2020, employing the search 
string "physical education" AND "selfassessment" within the fields "article," "keywords," and "abstract". Supplementary analysis of the reference lists of the extracted articles was carried out manually to find potential articles, not electronically identified.

Main outcome(s): Determination of selfassessment practices studied in Physical Education context. Identification of selfassessment uses and magnitude of the effects, in Physical Education context. Definition of a research agenda about use of self-assessment in Physical Education.

Quality assessment / Risk of bias analysis: Evaluation of each article is made using the inclusion and exclusion criteria, defined in the systematic review protocol, and validated by two reviewers (M.Â.F., A.A.) independently. A third reviewer (H.S.) makes an independent decision, if uncertainty exists. Foreseeing a diversity of study types collected, assessment of risk of bias is made by two independent researchers (P.R.N and M.Â.F.), using TREND scale, a 22 item checklist proposed for studies using nonrandomized designs, which include a defined intervention that is being studied and a research design that provides for an assessment of the efficacy or effectiveness of the intervention (Des Jarlais, D. C., Lyles, C., Crepaz, N., \& the Trend Group, 2004; Fuller et al, 2014).

Strategy of data synthesis: The information obtained in the systematic review is summarized according to the following categories of analysis: Author and Year, Country, Educational Setting, Type of paper, Methods and Instrument, Purpose, Outcomes, Comparison: Uses of selfassessment, Observed effects of selfassessment.

Subgroup analysis: No analysis.

Sensitivity analysis: No analysis.

Language: English.

Country(ies) involved: Portugal.
Keywords: Physical Education; selfassessment; co-participative assessment; formative assessment; self-regulation; metacognition.

Contributions of each author:

Author 1 - Paulo Renato Nobre - Head of the project, methodology, software, formal analysis, writing and revision.

Email: prnobre@fcdef.uc.pt

Author 2 - Miguel Ângelo Fachada Validation, formal analysis, writing, review and editing.

Email: miguelfachada@fcdef.uc.pt

Author 3 - Antero Abreu - Validation, formal analysis.

Email: anteroabreu@fcdef.uc.pt

Author 4 - Hugo Sarmento - Methodology, software, validation, formal analysis.

Email: hugo.sarmento@uc.pt

Author 5 - Fernanda M Silva - Writing, review and editing.

Email: f.m.a.s_298@hotmail.com

Author 6 - Carlos M Soares - Writing preparation, review and editing.

Email: solrach2o@gmail.com

Author 7 - Ana Luísa Cristovão - Formal analysis, writing preparation.

Email: luisa_27@live.com.pt

Author 8 - Ana Teixeira - Writing and supervision.

Email: ateixeira@fcdef.uc.pt

Author 9 - José Pedro Ferreira Methodology, software, formal analysis and supervision.

Email: jpferreira@fcdef.uc.pt 